\title{
Non-alcoholic Fatty Liver Disease (NAFLD) as a major predictor of gestational diabetes mellitus (GDM) and early pregnancy miscarriage: a Prospective Cohort Study
}

Iresha Koralagedara ${ }^{1}$, Janith Warnasekara ${ }^{2}$, Dayaratne Korale Gedara $^{3}$, Nirmani de Silva ${ }^{2}$, and Suneth Agampodi ${ }^{2}$

${ }^{1}$ Rajarata University of Sri Lanka Faculty of Medicine and Allied Sciences

${ }^{2}$ Rajarata University of Sri Lanka

${ }^{3}$ Teaching Hospital Anuradhapura

June 21, 2021

\begin{abstract}
Objective Determine the association of Non-alcoholic fatty liver disease(NAFLD) to the occurrence of GDM and miscarriages. Design Population-based prospective cohort study. Setting Anuradhapura district-Sri Lanka. Sample All pregnant women(gestational age $<12$ weeks) registered in the national pregnancy care programme( $>98 \%$ coverage) during July to September-2019, recruited in the Rajarata Pregnancy Cohort(RaPCo)(included 80\% of registered mothers). Method Clinical, biochemical parameters and ultrasound scan(USS) of liver were performed at the baseline to assess NAFLD. Excluding pregnant women who reported any type of hyperglycemia at the baseline, the cohort was followed up to assess the occurrence of GDM (using latest WHO criteria) during 24-28 weeks of gestation. Miscarriages were documented. Main outcome measures GDM and miscarriages. Results The prevalence of Fatty liver grade(FLG)-II and I was $14.2 \%(\mathrm{n}=90), 37 \%(\mathrm{n}=234)$, respectively. The incidence of GDM among the normoglycemic pregnant women with FLG-II, I, and 0 were 162.2, 43.9, 11 per 1000, respectively. After adjusting for age, BMI, and other known risk factors, women with FLG-II had a relative risk(RR) of $12.5(95 \% \mathrm{CI}$ 2.2-66.4) for developing GDM compared to those with FLG-0. Pregnant women with FLG-I-(RR=5.1,95\%CI-1.7-15.1) and II-(RR=8.4,95\% CI-2.6-27.1) had a very high risk of early pregnancy miscarriage compared to FLG-0. Conclusion FLG-2 is a significant risk factor for GDM and miscarriages. Incorporating pre-conceptional or early pregnancy identification of NAFLD using simple USS into routine care provision will enable early risk identification and appropriate action. Funding Accelerating Higher Education Expansion and Development(AHEAD) grant(World Bank-funded project through University grant commission-Sri Lanka) funded this study. Keywords-NAFLD, GDM, Pregnancy, Ultrasound-scan, Miscarriages
\end{abstract}

Non-alcoholic Fatty Liver Disease (NAFLD) as a major predictor of gestational diabetes mellitus (GDM) and early pregnancy miscarriage: a prospective Cohort Study

Iresha Koralegedara ${ }^{1,2}$, Janith Warnasekara ${ }^{1}$, Korale Gedara Dayaratne ${ }^{3}$, Farika Nirmani de Silva ${ }^{1}$, Suneth Agampodi $^{1}$

${ }^{1}$ Department of Anatomy, Faculty of Medicine and Allied Sciences, Rajarata University of Sri Lanka.

${ }^{2}$ Department of Community Medicine, Faculty of Medicine and Allied Sciences, Rajarata University of Sri Lanka.

3 Department of Radiology, Teaching Hospital Anuradhapura, Sri Lanka.

\section{Corresponding Author}


Iresha Koralegedara

Address: Department of Anatomy, Faculty of Medicine and Allied Sciences, Rajarata University of Sri Lanka, Saliyapura, Sri Lanka. 50008.

Telephone: +94717982664

Email: kis.koralegedara@gmail.com

Running title : Prospective cohort study on Association between NAFLD and GDM/ miscarriages.

Abstract Word Count -248

Objective

Determine the association of Non-alcoholic fatty liver disease(NAFLD) to the occurrence of GDM and miscarriages.

\section{Design}

Population-based prospective cohort study.

\section{Setting}

Anuradhapura district-Sri Lanka.

\section{Sample}

All pregnant women(gestational age $<12$ weeks) registered in the national pregnancy care programme( $>98 \%$ coverage) during July to September-2019, recruited in the Rajarata Pregnancy Cohort(RaPCo)(included $80 \%$ of registered mothers).

\section{Method}

Clinical, biochemical parameters and ultrasound scan(USS) of liver were performed at the baseline to assess NAFLD. Excluding pregnant women who reported any type of hyperglycemia at the baseline, the cohort was followed up to assess the occurrence of GDM (using latest WHO criteria) during 24-28 weeks of gestation. Miscarriages were documented.

\section{Main outcome measures}

GDM and miscarriages.

\section{Results}

The prevalence of Fatty liver grade(FLG)-II and I was $14.2 \%(\mathrm{n}=90), 37 \%(\mathrm{n}=234)$, respectively. The incidence of GDM among the normoglycemic pregnant women with FLG-II, I, and 0 were 162.2, 43.9, 11 per 1000, respectively. After adjusting for age, BMI, and other known risk factors, women with FLG-II had a relative risk(RR) of 12.5(95\%CI 2.2-66.4) for developing GDM compared to those with FLG-0. Pregnant women with FLG-I-(RR=5.1,95\% CI-1.7-15.1) and II-(RR=8.4,95\%CI-2.6-27.1) had a very high risk of early pregnancy miscarriage compared to FLG-0.

\section{Conclusion}

FLG-2 is a significant risk factor for GDM and miscarriages. Incorporating pre-conceptional or early pregnancy identification of NAFLD using simple USS into routine care provision will enable early risk identification and appropriate action.

\section{Funding}

Accelerating Higher Education Expansion and Development(AHEAD) grant(World Bank-funded project through University grant commission-Sri Lanka) funded this study. 
Keywords- NAFLD, GDM, Pregnancy, Ultrasound-scan, Miscarriages

\section{Tweetable abstract}

Pregnant women with fatty liver and without previous hyperglycemia have 12 times the risk of developing gestational diabetes mellitus (GDM) than those without fatty liver. Similarly, the risk of miscarriage is around eight times higher among pregnant women with a fatty liver.

Full text - (Word count -3484; excluding titles, subtitles, table headings and figure legends)

\section{Introduction}

Non-alcoholic fatty liver disease (NAFLD) is an initial manifestation of various pathological conditions, such as non-alcoholic steatohepatitis, cirrhosis and, liver malignancies ${ }^{1}$. The most implicated mechanism for NAFLD is insulin resistance ${ }^{2}$. Supra-physiological levels of glucose, sucrose, and, fructose can induce lipogenic genes through various mechanisms that lead to de novo lipogenesis and inhibit fatty acid oxidation causing deposition of fatty acids in various organs such as liver ${ }^{3}$. Latter combined insulin resistance, hormones secreted by the adipocytes, nutritional factors, gut microbiota and, genetic factors to describe the pathogenesis of NAFLD. The pooled regional incidence of NAFLD in Asian countries is 52 per 1,000 person-years compared to 28 per 1,000 person-years in the $\mathrm{West}^{4}$. The prevalence of lean NAFLD in Asia is $19 \%$, while it is $7 \%$ in the United States ${ }^{5}$.

While NAFLD is the commonest liver disease in the west, its effect on pregnancy has not been discussed widely until recently ${ }^{6}$. Early retrospective studies have reported a low prevalence (28.9/100,000 per pregnancies) on NAFLD ${ }^{7}$, yet with definitive adverse pregnancy outcomes. Recent studies show a varying degree of NAFLD among pregnant women, with $15 \%$ in Canada ${ }^{8}, 14.3 \%-16.7 \%$ in USA ${ }^{9,10}, 18.4 \%$ in Korea ${ }^{11}$, and $18.2 \%$ in Sri Lanka ${ }^{12}$. The secondary data analysis of US inpatient sample of 18,574,225 pregnancies shows that the prevalence of NAFLD after 20 weeks of gestation has tripled over a period of 10 years ${ }^{13}$. Since its first report in 2011, NAFLD is identified as a major predictor of many fetal and maternal adverse outcomes including miscarriages ${ }^{14}$, gestational diabetes mellitus (GDM) ${ }^{11,15-18}$, hypertensive complications ${ }^{19,20}$, higher caesarean sections ${ }^{20}$, intrahepatic cholestasis in pregnancy ${ }^{21}$, pre-term birth ${ }^{19}$, low birth weight ${ }^{21}$ and postpartum haemorrhage ${ }^{7}$.

Being an insulin-resistant state, pregnancy itself has a higher risk for NAFLD as well as developing hyperglycaemia. Thus, one of the main adverse pregnancy outcomes associated with NAFLD is GDM. The pooled global prevalence of GDM using IADPSG criteria is reported as $10.6 \%\left(95 \%\right.$ CI $10.5-10.6 \%{ }^{22}$ whereas the estimates for 2005-2015 shows a wide disparity across WHO regions ranging from $5.8 \%$ in Europe to 12.9 in middle East and north Africa. The incidence of GDM among pregnant women with NAFLD was shown to be more than $20 \%^{23}$. and the severity of NAFLD is proportional to the risk of GDM and large for gestational age (LGA) babies ${ }^{24}$. The unconfounded effect of NAFLD on GDM was estimated with an odds ratio around two in two prospective cohort studies; one with OR 2.50 (95\% CI 1.07, 5.77) ${ }^{23}$ and another with an OR $2.2(95 \% \text { CI: } 1.1-4.3)^{25}$ and 6.5, (95\% CI: 2.3-18.5) in another study. While early pregnancy NAFLD is almost established as a major predictor of GDM, only a limited number of prospective studies are available in global literature and none from South Asian region, a region having high incidence of both NAFLD and GDM. According to our knowledge none of prospective studies are available globally about the association between NAFLD and miscarriages. The purpose of the present study was to determine the role of NAFLD as a risk factor for GDM and early pregnancy miscarriages among Sri Lankan pregnant women.

\section{Methodology}

\section{Study design and setting}

This study was carried out as a part of a large population-based prospective cohort study: the Rajarata Pregnancy Cohort (RaPCo). The full study design of RaPCo is published elsewhere ${ }^{26}$. The study was carried out in the Anuradhapura district, which is the largest district in Sri Lanka. The resident population in the district is 929,539 , and in 2019, 15,811 pregnant women were registered with the national maternal care 
programme. Of them, 12,984 were registered in field clinics before 8 weeks of gestation, 2063 were registered in field clinic visits at 8-12 weeks of gestation, and $98.6 \%$ had at least one clinic visit before delivery ${ }^{27}$.

\section{Baseline assessment}

\section{Figure 1 - Summary of the methodology}

All pregnant women with a period of gestation of less than 12 weeks who registered with public health midwives from early July-end of September 2019 were recruited from all MOH areas in Anuradhapura district for the study. Collection of socio-demographic data was performed by medical undergraduates in the third year of training, and clinical data collection and the examination were performed by MBBS-qualified medical officers. Baseline data was collected by using an interviewer-administered questionnaire. Pretested interviewer administered questionnaire was used for data collection on sociodemographic, obstetric, gynecological and medical backgrounds. Those with a history of diabetes mellitus, uncertain period of amenorrhea (POA), known liver diseases (except NAFLD) and history of using steatogenic drugs were excluded from the initial recruitment.

Clinical examination was performed to elicit any signs of pre-existing diseases. Blood pressure was recorded using a high precision automated blood pressure measuring instrument (Omron Corporation) as the mean of two readings taken 5 min apart from both arms it and was categorized as normal and high values for the first and second trimesters according to the NICE guidelines $2019{ }^{28}$. Height (Ht), weight (Wt), body mass index (BMI), waist circumference (WC), hip circumference (HC) and waist to hip ratio (WHR) was taken as anthropometric measurements.

Weight was measured by using a digital weighing scale and height was measured by using a portable stadiometer. According to standard formula of BMI, categorized as overweight, obesity and morbid obesity and according to standard cutoff levels for Asian population by WHO guidelines. Waist circumference (WC) was measured by placing a non-stretchable fibre-glass measuring tape around the waist midway between the last rib and iliac crest with the subject in the standing position. Two measurements were taken for improvement of the accuracy of measurement. Hip circumference $(\mathrm{HC})$ was measured the maximum circumference of buttocks. Two measurements were taken and sum of the measurement will be taken as WC and HC. Some data was obtained from maternal pregnancy record. This entered data was standardized to make sure the routine data are high quality ${ }^{29}$. During first trimester of pregnancy there are very minimal changes of weight gain, and changes of waist circumference. Therefore we use standard calculation methods and ranges for Asian adult population to calculate BMI, cut off levels of obesity, waist circumference pregnancy period.

Baseline investigations were done to identify common metabolic complications and to exclude any pathological conditions not related to NAFLD. These investigations include routine basic investigations of mothers should be done their antenatal period and investigations for diagnose dyslipidemia and liver related disorders. Blood samples were collected by using standard guidelines by well qualified public health nursing sisters. Pre requisites for sample collection were informed to mothers prior to the blood collection date. Venipuncture was done at ante-cubital fossa under aseptic conditions and universal precautions. Internal quality control was performed before each and every analytical run. Peer group comparison was done every month during sample collection and period of analysis of collected blood samples.

An oral glucose tolerance test (OGTT) was performed on all participants at the recruitment and second trimester. Diagnoses of diabetes mellitus in pregnancy (DIP) and gestational diabetes mellitus (GDM) were performed using WHO (2016) criteria ${ }^{30}$. GDM was defined as fasting blood sugar (FBS) of 92-125 mg/dl and/or $2^{\text {nd }}$-hour plasma glucose of $153-199 \mathrm{mg} / \mathrm{dl}$ by a 75 -g glucose test anytime in pregnancy. Those with FBS [?] $126 \mathrm{mg} / \mathrm{dl}$ and/or $2^{\text {nd }}$-hour plasma glucose [?] $200 \mathrm{mg} / \mathrm{dl}$ were labelled as having diabetes mellitus in pregnancy ${ }^{30}$. Level of serum aminotransferaces, gamma glutamyl transferaces and serum lipid level was done as baseline screening tests to identify any liver related pathological conditions and pre-existing dyslipidemia.

After obtaining written informed consent, all eligible study participants were offered an ultrasound scan 
(USS) abdomen. USS was performed by competent and qualified investigators. Liver echogenicity was compared with the ipsilateral renal cortex and the spleen, and the attenuation of waves, loss of demarcation of the diaphragm and poor demarcation of the intrahepatic architecture were examined. Thus, fatty liver was graded as ${ }^{20}$; Grade 0: Normal liver echogenicity, Grade I: Diffusely increased hepatic echogenicity but periportal and diaphragmatic echogenicity is still appreciable, Grade II: Diffusely increased hepatic echogenicity obscuring periportal echogenicity but diaphragmatic echogenicity is still appreciable, and Grade III: Diffusely increased hepatic echogenicity obscuring periportal and diaphragmatic echogenicity.

Although comparison of fatty liver between pregnancy and the general population is not a major objective of the study, a small sample of an age-matched randomly selected females was subjected to USS to overcome the possible bias of early pregnancy liver changes.

\section{Follow-up}

After excluding those with GDM and DIP based on the baseline OGTT, all pregnant women recruited were followed up. OGTT was repeated in between 24 and 28 weeks of POA.

\section{Diagnosis and confirmation of miscarriage}

All the miscarriages occurred before 24 weeks of POA and diagnosed by consultant obstetrics and gynecologist or consultant radiologist through routine health care system were confirmed as the miscarriages.

\section{Data analysis}

All continuous variables were summarized as the means with standard deviations according to the fatty liver grade. The discrete data were presented as medians. To determine the association between fatty liver grade and a high OGTT value, binary logistic regression was performed by considering a high or normal OGTT value as the binary dependent variable. Adjusted odds ratios were calculated for the risk factors.

\section{Results}

\section{Baseline assessment}

A total of 634 pregnant women with POA [?]12 weeks were recruited from 254 small health administrative areas (PHM areas) representing all $22 \mathrm{MOH}$ divisions. The mean age of the sample was 28.5 (SD 5.8) years, and most of them (55.2\%) were in the age category of 21-30 years (Table 1 ). The majority (31.5\%) of the mothers were in their second pregnancy and had completed post-primary education.

Table 1: Baseline characteristics of the 634 first trimester pregnant women recruited for NAFLD assessment

Though the diagnosis of diabetes mellitus reported by only 6 women, 18 (including the 6 with DM) reported history of hyperglycemia detected once or more than once. Prevalence of hypertension $(36,5.9 \%)$, dyslipidemias $(8,1.3 \%)$, hypothyroidism $(17,2.7 \%)$ was low. Of the multigravida women, history of GDM was reported by $17(2.7 \%)$ and the history of having at least one miscarriage was $19.1 \%(\mathrm{n}=121)$.

Of the pregnant women recruited, $324(51.2 \%)$ had either grade I fatty liver (FLG I) ( $\mathrm{n}=234,37.0 \%, 95 \%$ CI $33.2 \%-40.9 \%$ ) or grade II fatty liver (FLG II) $(90,14.2 \%, 95 \%$ CI $11.6 \%-17.2 \%)$. None of the participants had fatty liver grade III (FLG III).

The prevalence of fatty liver in the non-pregnant group was $51.7 \%$, with grade II and grade I prevalence of $14.2 \%$ (n 21) and 37.0\%(n 4), respectively. There was no statistically significant difference between the two groups.

All tested liver parameters, biophysical parameters and OGTT $2^{\text {nd }}$ hour values gradually increased across the fatty liver grades. The difference between fatty liver grades I and II was higher than the difference between grades 0 and I in all parameters except the FBS value, minimum DBP, portal vein diameter and dome-to-pole length. FBS in the first trimester was reduced gradually across the fatty liver grades (Table 2). 
Table 2: Comparison of liver parameters, plasma glucose and biophysical measurements by fatty liver grades among 632 first trimester pregnant women

Of the participants recruited, 84 were excluded from the follow-up study due to detection of DIP (13, $2.1 \%)$, GDM $(66,10.4 \%)$ and missing OGTT values $(5,0.8 \%)$. Of the 548 eligible participants, $17(3.1 \%)$ pregnant women left the area, and from the rest, $27(5.1 \%)$ had late first-trimester or second trimester miscarriages.(Figure 2)

\section{Figure 2 - Study flow chart of the participants recruited and loss to follow up}

The cumulative incidences of miscarriages among fatty liver grades 0 , I and II were 14.9, 75.8 and 125.0 per 1000 pregnancies, respectively. Compared to those with FLG 0, pregnant women with FLG I had more than 5 times the risk (RR 5.1, 95\% CI 1.7-15.1) of miscarriage. Similarly, FLG II had more than 8 times the risk of miscarriage (8.4, 95\% CI 2.6-27.1) compared with FLG 0. (Table 3)

Table 3: Incidence and relative risk of developing GDM/DM at the second trimester by fatty liver grade

Of the rest, 348 pregnant women were assessed at the end of the second trimester. Two cases of DM in pregnancy and 11 GDM cases were detected among previously normoglycaemic women. The incidence of DIP/GDM of FLG I, II and III in the second trimester was 11.0, 43.9 and 162.2 per 1000 pregnancies respectively. The unadjusted relative risk of developing DIP/GDM in the second trimester compared to FLG 0 was 3.8 (95\% CI 0.79-19.4) and 12.5 (95\% CI 2.6-60.0) for FLG I and FLG II, respectively. The unconfounded effect of FL on developing GDM/DIP was assessed using a binary logistic regression model with waist-to-hip ratio, age, parity, FL grades, family history of diabetes, female education, and BMI as probable confounders (Table 4). Only the FL grade II was emerged as a significant risk factor for developing DIP/GDM with a OR of 12.3 .

Table 4: Risk factors for developing GDM/DM in pregnancy

\section{Discussion}

\section{Main Findings}

NAFLD during pregnancy partially reflects the physiological changes with fluctuation of oestrogen, lipid levels and rapid weight gain during pregnancy ${ }^{31}$. Nevertheless, the link between NAFLD and GDM has been studied on both directions. NAFLD in the first trimester has shown to be a risk factor for dysglycemia in mid pregnancy ${ }^{32}$, and having gestational diabetes was also identified as a risk factor for postpartum development of NAFLD ${ }^{33}$. This study indicates that prevalence of fatty liver among pregnant women in Anuradhapura, Sri Lanka is much higher than the reported values from HICs and the previously reported in the South Asian context. Strengthening the evidence generated elsewhere ${ }^{34,35}$ this prospective study indicate that NAFLD, specifically Fatty liver Grade II? Correct? is a major risk factor for subsequent development of GDM in pregnancy. Although this association is reported in HICs, this is the first study to reflect this in the rural Asian population using a community based on a prospective cohort study. The study also depict that NAFLD is associated with early pregnancy miscarriage. Several past studies report that NAFLD may not be a significant risk factor for diabetes after adjusting for BMI and age ${ }^{32}$. In our study, the fatty liver grade II was the only risk factor for developing GDM/DIP, even after adjusting for these confounding variables. The diagnostic thresholds and criteria for GDM are different across the globe and are evolving. The strength of the association in this study is difficult to compare directly with many previous studies due to the differences in GDM diagnostic criteria. De Souza et al. used similar criteria for GDM and revealed an adjusted OR ranging from 6.8-7.8 ${ }^{34}$. Mousa et al. also reported significantly higher incidence of GDM among pregnant mothers with fatty liver ${ }^{32}$. Our estimates are much larger yet have wide confidence intervals (similar to the study by De Souza et al.)

\section{Strengths}


This study carries several strengths. The study design is community based and systematically represent more than $80 \%$ of pregnant women newly registered in Anuradhapura, the largest district of Sri Lanka. The study represent a rural population. The results depict follow up of a cohort of pregnant women. Hence the prospective nature implies in prediction of the direction of the association between NAFLD and GDM. We have used the latest WHO criteria for the assessment of GDM. The study has clearly excluded pregnant women who have had all types of hyperglycaemia. Hence the clarity of the association is strong. In addition identification of NAFLD and GDM were performed using measurements adopted to standard protocols.

\section{Interpretations}

The observed NAFLD prevalence of $51.3 \%$ in early pregnancy seems higher than the previously reported values for Sri Lanka $(18.2 \%)^{20}$, Canada $(17.6 \%)^{25}$, Korea $(18.4 \%)^{11,38}$, and USA $(14.3-16.7 \%)^{10}$. However, the prevalence in this study is not significantly different from the comparison group of non-pregnant reproductive age women from same geographical location. Irrespective of pregnancy, this high prevalence of fatty liver in this rural young woman (mean age 28.8 years) needs to be taken seriously as a major predicter of future NCDs.

NAFLD is considered as a hepatic manifestation of metabolic syndrome ${ }^{39}$. According to the multiple hit hypothesis proposed by Elenza et al. increasing oxidative stress, inflammation, endothelial dysfunction can lead to development of NAFLD ${ }^{40}$. These underlying issues leading to NAFLD may have other effects on the mother and child which has not been fully evaluated yet. One of the most important findings of this study was the unprecedented observation of FL as a major risk factor for mid-pregnancy miscarriages with an odds ratio of 8.4. Liu $\mathrm{Y}$ et al. showed that abortions in women with NAFLD were higher than those without NAFLD ( $72.4 \%$ vs. $69.3 \%$, age-adjusted $\mathrm{P}=0.001){ }^{41}$. The pathophysiology behind this association is still not very clear. However, we can assume that the oxidative stress, endothelial damage and inflammation may predispose to development of early pregnancy loss ${ }^{40}$. This observation with high effect size is of significant public health importance in predicting mid-trimester miscarriages and probably formulating new guidelines for pregnancy care programmes.

Current evidence on diabetes clearly shows that South Asians are at an increased risk of all metabolic derangements, including NAFLD, DM and GDM ${ }^{42}$. Combining this knowledge with public health service provisions could be a practical and comprehensive approach in pregnancy care programmes. USS has been used for many years for pregnancy care, especially in the first trimester, as a "dating scan". A simple added step in dating scans to screen for fatty liver during pregnancy may show the risk of developing hyperglycaemia and mid-pregnancy miscarriage. Our study shows that NAFLD is a better predictor of these conditions than the traditional "risk factors" considered in pregnancy. Beyond pregnancy, the diagnosis of NAFLD in pregnant women would be an early life opportunity to screen for fatty liver to prevent future non-communicable diseases.

\section{Limitations}

In this study we used USS to diagnose NAFLD instead of the gold standard liver biopsy. USS is a noninvasive, acceptable and feasible alternative method, especially in pregnant women. The sensitivity of the diagnosis of fatty liver in ultrasound scans ranges from $60-94 \%$, and the specificity is from $84-95 \%$. The sensitivity of detecting fatty liver is increased when the degree of fatty liver is increased. In morbid obesity, sensitivity and specificity are reduced from $49-75 \%$ due to technical errors. However, evidence shows that the reliability of USS for diagnosing fatty liver is higher than that of histology in people with moderate and severe fatty liver. In addition, the grading of fatty liver is subjective and operator-dependent ${ }^{12}$. The objective of the FL scan in our study is risk prediction rather than a precise diagnosis of steatosis, and for that purpose, routine USS will be adequate.

The wide confidence intervals are due to the relatively low number of GDM reported show the need for a study with a larger sample size for better estimates of risk. Application of latest WHO criteria ${ }^{37}$ led to exclusion of 66 pregnant women from the baseline assessment whom should not be excluded if the previous classification was used. 


\section{Conclusion}

Fatty liver grade 2, diagnosed by a simple USS is a major risk factor for GDM and early pregnancy miscarriage. The prevalence of NAFLD is higher than that is reported elsewhere and in the same context in the past. Pre-conceptional and/or early pregnancy diagnosis of NAFLD probably incorporated to the routine USS in pregnancy should be considered for the early identification of this important risk factor. We recommend to incorporation of USS scan to detect fatty liver with the routine dating scan of pregnancy so that additional preventive health care could be provided for the mothers having grade 2 fatty liver.

\section{Acknowledgement}

We acknowledge all participated mothers and all data collectors throughout the study. This study is conducted as a part of a large cohort study (RaPCo). We acknowledge all the other investigators who involved in RaPCo cohort study.

\section{Declaration of interest}

All the authors declare that there is no conflict of interest.

\section{Authors Contributions}

Conceptualization, designing the study and data analysis was done by IK, JW, and SA. IK, KD, JW and FN did the data curation while SA did funding acquisition. Experiment procedures were done by IK, KD and FN. All authors involved in writing the Article while SA and KD involved in supervision.

\section{Ethical considerations}

The Ethical clearance was obtained from the Ethics review committee of Faculty of Medicine and Allied Sciences, Rajarata University of Sri Lanka under ERC/2019/22 and approved on 15/08/2019. Written informed consent was taken from all pregnant mothers to participate in this study as well as to use routinely collected data for this research purpose. The participants were informed that this research is conducted in parallel with the routine maternal and child health service and any "abnormality" will be reported to the health provider with the participant's consent. In addition, consent had been sought for use of serum sample for screening of diabetes mellitus, liver biochemistry and serum cholesterol level and any other future studies, that may require baseline assessment of serum. Data was linked to the health records using a classified code, so that the confidentiality of data secured. Individual level data will not be presented in any form and summary data was used in research publications and data dissemination.

\section{Role of the funding source}

This study was funded through the Accelerating Higher Education Expansion and Development (AHEAD) grant, a World Bank funded project through University grant commission Sri Lanka. The funding agency has no role in the design of the study and collection, analysis, and interpretation of data and in writing the manuscript.

\section{References}

1. Younossi Z, Anstee QM, Marietti M, Hardy T, Henry L, Eslam M, et al. Global burden of NAFLD and NASH: trends, predictions, risk factors and prevention. Nat Rev Gastroenterol Hepatol [Internet]. 2018 Jan 20 [cited 2019 Aug 30];15(1):11-20. Available from: http://www.ncbi.nlm.nih.gov/pubmed/28930295

2. Basaranoglu M, Neuschwander-Tetri BA. Nonalcoholic Fatty Liver Disease: Clinical Features and Pathogenesis. Gastroenterol Hepatol (N Y) [Internet]. 2006 Apr [cited 2019 Feb 24];2(4):282-91. Available from: http://www.ncbi.nlm.nih.gov/pubmed/28286458

3. Prins GH, Luangmonkong T, Oosterhuis D, Mutsaers HAM, Dekker FJ, Olinga P. A pathophysiological model of non-alcoholic fatty liver disease using precision-cut liver slices. Nutrients [Internet]. 2019 Mar 1 [cited 2021 Mar 20];11(3). Available from: https://pubmed.ncbi.nlm.nih.gov/30818824/ 
4. Vernon G, Baranova A, Younossi ZM. Systematic review: the epidemiology and natural history of non-alcoholic fatty liver disease and non-alcoholic steatohepatitis in adults. Aliment Pharmacol Ther [Internet]. 2011 Aug 1 [cited 2019 Feb 22];34(3):274-85. Available from: http://doi.wiley.com/10.1111/j.13652036.2011.04724.x

5. VanWagner LB, Armstrong MJ. Lean NAFLD: A not so benign condition? Hepatol Commun [Internet]. 2018 [cited 2019 Feb 22];2(1):5-8. Available from: http://www.ncbi.nlm.nih.gov/pubmed/29404505

6. Page LM, Girling JC. A novel cause for abnormal liver function tests in pregnancy and the puerperium: Non-alcoholic fatty liver disease. BJOG An Int J Obstet Gynaecol [Internet]. 2011 Nov 1 [cited 2021 May 29];118(12):1532-5. Available from: https://obgyn.onlinelibrary.wiley.com/doi/full/10.1111/j.14710528.2011.03070.x

7. McIntyre HD, Catalano P, Zhang C, Desoye G, Mathiesen ER, Damm P. Gestational diabetes mellitus [Internet]. Vol. 5, Nature Reviews Disease Primers. Nature Publishing Group; 2019 [cited 2021 Mar 21]. p. 1-19. Available from: https://www.nature.com/articles/s41572-019-0098-8

8. De Souza LR, Berger H, Retnakaran R, Vlachou PA, Maguire JL, Nathens AB, et al. Nonalcoholic fatty liver disease in early pregnancy predicts dysglycemia in mid-pregnancy: prospective study. Am J Gastroenterol [Internet]. 2016 May 1 [cited 2021 Apr 28];111(5):665-70. Available from: https://pubmed.ncbi.nlm.nih.gov/26977755/

9. Sattari M, Bril F, Egerman R, Kalavalapalli S, Cusi K. Relationship between non-alcoholic fatty liver disease during pregnancy and abnormal glucose metabolism during and after pregnancy. J Investig Med [Internet]. 2020 Mar 1 [cited 2021 May 29];68(3):743-7. Available from: https://pubmed.ncbi.nlm.nih.gov/31852748/

10. Sarkar M, Djerboua M, Flemming JA. NAFLD Cirrhosis Is Rising Among Childbearing Women and Is the Most Common Cause of Cirrhosis in Pregnancy. Clin Gastroenterol Hepatol [Internet]. 2021 Jan [cited 2021 May 29]; Available from: https://pubmed.ncbi.nlm.nih.gov/33465483/

11. Lee SM, Kwak SH, Koo JN, Oh IH, Kwon JE, Kim BJ, et al. Non-alcoholic fatty liver disease in the first trimester and subsequent development of gestational diabetes mellitus. Diabetologia [Internet]. 2018 Nov 23 [cited 2019 Jan 3];1-11. Available from: http://link.springer.com/10.1007/s00125-018-4779-8

12. HMM H, I K, TP W, G L. Prevalence and Associations of Non-Alcoholic Fatty Liver Disease (NAFLD) in Sri Lankan Patients With Type 2 Diabetes: A Single Center Study. Diabetes Metab Syndr. 2019;13(1).

13. Sarkar M, Grab J, Dodge JL, Gunderson EP, Rubin J, Irani RA, et al. Non-alcoholic fatty liver disease in pregnancy is associated with adverse maternal and perinatal outcomes. J Hepatol. 2020 Sep 1;73(3):516-22.

14. Liu Y, Lu J, Xu M, Xu Y, Li M, Wang T, et al. Association between history of abortion and nonalcoholic fatty liver disease in middle-aged and elderly Chinese women. Ann Epidemiol. 2013 Mar;23(3):119-23.

15. Ajmera VH, Gunderson EP, VanWagner LB, Lewis CE, Carr JJ, Terrault NA. Gestational Diabetes Mellitus Is Strongly Associated With Non-Alcoholic Fatty Liver Disease. Am J Gastroenterol [Internet]. 2016 May 22 [cited 2019 Jan 30];111(5):658-64. Available from: http://www.ncbi.nlm.nih.gov/pubmed/27002796

16. Foghsgaard S, Andreasen C, Vedtofte L, Andersen ES, Bahne E, Strandberg C, et al. Nonalcoholic Fatty Liver Disease Is Prevalent in Women With Prior Gestational Diabetes Mellitus and Independently Associated With Insulin Resistance and Waist Circumference. Diabetes Care [Internet]. 2017 [cited 2019 Jan 30];40:109. Available from: http://www.diabetesjournals

17. The Role of Non-Alcoholic Fatty Liver Disease in Gestational Diabetes - Full Text View - ClinicalTrials.gov [Internet]. [cited 2019 Jan 30]. Available from: https://clinicaltrials.gov/ct2/show/NCT01855386

18. Ajmera VH, Gunderson EP, VanWagner LB, Lewis CE, Carr JJ, Terrault NA. Gestational Diabetes Mellitus Is Strongly Associated With Non-Alcoholic Fatty Liver Disease. Am J Gastroenterol [Internet]. 2016 
May 22 [cited 2019 Jan 28];111(5):658-64. Available from: http://www.ncbi.nlm.nih.gov/pubmed/27002796

19. NAFLD in pregnancy increases risks for mother and baby - EASL-The Home of Hepatology. [Internet]. [cited 2021 Feb 8]. Available from: https://easl.eu/press-release/nafld-pregnancy-risks/

20. Herath HMM, Kodikara I, Weerarathna TP, Liyanage G. Prevalence and associations of non-alcoholic fatty liver disease (NAFLD) in Sri Lankan patients with type 2 diabetes: A single center study. Diabetes Metab Syndr Clin Res Rev [Internet]. 2019 Jan [cited 2019 Jan 31];13(1):246-50. Available from: http://www.ncbi.nlm.nih.gov/pubmed/30641706

21. Rathi U, Bapat M, Rathi P, Abraham P. Effect of liver disease on maternal and fetal outcome-a prospective study. Indian J Gastroenterol [Internet]. [cited 2019 Mar 8];26(2):59-63. Available from: http://www.ncbi.nlm.nih.gov/pubmed/17558066

22. Behboudi-Gandevani S, Amiri M, Bidhendi Yarandi R, Ramezani Tehrani F. The impact of diagnostic criteria for gestational diabetes on its prevalence: A systematic review and meta-analysis [Internet]. Vol. 11, Diabetology and Metabolic Syndrome. BioMed Central Ltd.; 2019 [cited 2021 May 29]. p. 11. Available from: https://doi.org/10.1186/s13098-019-0406-1

23. SM L, SH K, JN K, IH O, JE K, BJ K, et al. Non-alcoholic Fatty Liver Disease in the First Trimester and Subsequent Development of Gestational Diabetes Mellitus. Diabetologia. 2019;62(2).

24. Mi Lee S, Shin Park J, Norwitz ER, Nam Koo J, Hwan Oh I, Jae Kim B, et al. Nonalcoholic fatty liver disease in the first trimester as a risk factor for large-for-gestational-age birthweight: a prospective study [Internet]. Vol. 218, American Journal of Obstetrics and Gynecology. 2018 [cited 2019 Jan 30]. Available from: https://www.ajog.org/article/S0002-9378(17)32087-2/pdf

25. De Souza LR, Berger H, Retnakaran R, Vlachou PA, Maguire JL, Nathens AB, et al. NonAlcoholic Fatty Liver Disease in Early Pregnancy Predicts Dysglycemia in Mid-Pregnancy: Prospective Study. Am J Gastroenterol [Internet]. 2016 May 15 [cited 2019 Jan 28];111(5):665-70. Available from: http://www.ncbi.nlm.nih.gov/pubmed/26977755

26. Agampodi TC, Wickramasinghe ND, Prasanna RIR, Irangani MKL, Banda JMS, Jayathilake PMB, et al. The Rajarata Pregnancy Cohort (RaPCo): study protocol. BMC Pregnancy Childbirth. 2020 Dec;20(1):374.

27. MOMCH Unit, Regional Director of Health services, Anuradhapura SL. Annual Statistics. 2019.

28. Overview | Hypertension in pregnancy: diagnosis and management | Guidance | NICE. [cited 2019 Apr 26]; Available from: https://www.nice.org.uk/guidance/cg107

29. (PDF) Management of obesity [Internet]. [cited 2019 Mar 9]. Available from: https://www.researchgate.net/publication/301302217_Management_of_obesity

30. WHO recommendations on antenatal care for a positive pregnancy experience.

31. Lao TT. Implications of abnormal liver function in pregnancy and non-alcoholic fatty liver disease [Internet]. Vol. 68, Best Practice and Research: Clinical Obstetrics and Gynaecology. Bailliere Tindall Ltd; 2020 [cited 2021 May 28]. p. 2-11. Available from: https://pubmed.ncbi.nlm.nih.gov/32312689/

32. Mousa N, Abdel-Razik A, Shams M, Sheta T, Zakaria S, Shabana W, et al. Impact of non-alcoholic fatty liver disease on pregnancy. Br J Biomed Sci [Internet]. 2018 Oct 2 [cited 2019 Jan 28];75(4):197-9. Available from: http://www.ncbi.nlm.nih.gov/pubmed/29943682

33. Le MH, Devaki P, Ha NB, Jun DW, Te HS, Cheung RC, et al. Prevalence of non-alcoholic fatty liver disease and risk factors for advanced fibrosis and mortality in the United States. Yu ML, editor. PLoS One [Internet]. 2017 Mar 27 [cited 2019 Feb 22];12(3):e0173499. Available from: http://dx.plos.org/10.1371/journal.pone.0173499 
34. De Souza LR, Berger H, Retnakaran R, Vlachou PA, Maguire JL, Nathens AB, et al. Hepatic fat and abdominal adiposity in early pregnancy together predict impaired glucose homeostasis in mid-pregnancy. Nutr Diabetes. 2016;6(9):e229.

35. Herath RP, Siriwardana SR, Ekanayake CD, Abeysekara V, Kodithuwakku SUA, Herath HP. Nonalcoholic fatty liver disease and pregnancy complications among Sri Lankan women: A cross sectional analytical study. Peterson JM, editor. PLoS One [Internet]. 2019 Apr 12 [cited 2020 Dec 1];14(4):e0215326. Available from: https://dx.plos.org/10.1371/journal.pone.0215326

36. Dahanayaka N, Agampodi S, Ranasinghe O, Jayaweera P, Wickramasinghe W, Adhikari A, et al. Inadequacy of the risk factor based approach to detect gestational diabetes mellitus. Ceylon Med J [Internet]. 2012 Mar 25 [cited 2019 Mar 30];57(1):5. Available from: https://cmj.sljol.info/article/10.4038/cmj.v57i1.4193/

37. International Association of Diabetes and Pregnancy Study Groups Consensus Panel M, Metzger BE, Gabbe SG, Persson B, Buchanan TA, Catalano PA, et al. International association of diabetes and pregnancy study groups recommendations on the diagnosis and classification of hyperglycemia in pregnancy. Diabetes Care. 2010 Mar;33(3):676-82.

38. Hagström H, Höijer J, Ludvigsson JF, Bottai M, Ekbom A, Hultcrantz R, et al. Adverse outcomes of pregnancy in women with non-alcoholic fatty liver disease. Liver Int [Internet]. 2016 Feb [cited 2019 Jan 29];36(2):268-74. Available from: http://www.ncbi.nlm.nih.gov/pubmed/26114995

39. Gastaldelli A. Fatty liver disease: The hepatic manifestation of metabolic syndrome [Internet]. Vol. 33, Hypertension Research. Nature Publishing Group; 2010 [cited 2021 Apr 29]. p. 546-7. Available from: www.nature.com/hr

40. Buzzetti E, Pinzani M, Tsochatzis EA. The multiple-hit pathogenesis of non-alcoholic fatty liver disease (NAFLD). Metabolism [Internet]. 2016 Aug [cited 2019 Feb 24];65(8):1038-48. Available from: http://www.ncbi.nlm.nih.gov/pubmed/26823198

41. Liu Y, Lu J, Xu M, Xu Y, Li M, Wang T, et al. Association between history of abortion and nonalcoholic fatty liver disease in middle-aged and elderly Chinese women. Ann Epidemiol [Internet]. 2013 Mar [cited 2021 Mar 9];23(3):119-23. Available from: https://pubmed.ncbi.nlm.nih.gov/23298439/

42. Misra A, Khurana L. The metabolic syndrome in South Asians: Epidemiology, determinants, and prevention. Metabolic Syndrome and Related Disorders. 2009.

\section{Figure Legends}

Figure 1 - Summary of the methodology

Figure 2 - Study flow chart of the participants recruited and loss to follow up

Table 2: Comparison of liver parameters, plasma glucose and biophysical measurements by fatty liver grades among 634 first trimester pregnant women

\begin{tabular}{lllll}
\hline Variable & Fatty Liver Grade & Fatty Liver Grade & Fatty Liver Grade & Fatty Liv \\
& & $\mathbf{0}(\mathbf{n}=\mathbf{3 0 8})$ & & $\mathbf{N}$ \\
& $\mathbf{N}$ & $\mathbf{M e a n}$ & $\mathrm{SD}$ & 185 \\
Age (Years) & 269 & 27.8 & 5.7 & 198 \\
Liver Parameters & & & 1.15 \\
Dome-to-pole length (cm) & 274 & 12.7 & 0.13 & 131 \\
Portal vein diameter (mm) & 263 & 1.06 & 5 & 133 \\
AST & 193 & 17 & 7 & 132 \\
ALT & 194 & 16 & 7 &
\end{tabular}




\begin{tabular}{lllll}
\hline Variable & Fatty Liver Grade & Fatty Liver Grade & Fatty Liver Grade & Fatty Liv \\
\hline FBS & 280 & 76.8 & 6.5 & 203 \\
OGTT 2 $^{\text {nd }}$ hour & 280 & 103.4 & 18.3 & 203 \\
Biophysical Measurements & & & 194 \\
Min. systolic BP (Hg mm) & 272 & 101 & 8 & 194 \\
Min. diastolic BP (Hg mm) & 272 & 64 & 4.2 & 188 \\
BMI & 260 & 21.9 & 0.07 & 181 \\
Waist-to-hip ratio & 261 & 0.82 &
\end{tabular}

Table 3: Incidence and relative risk of developing GDM/DM at the second trimester by fatty liver grade

\begin{tabular}{lllll}
\hline Fatty Liver Grade & New Cases & $\begin{array}{l}\text { Total No. Followed } \\
\text { Up }\end{array}$ & $\begin{array}{l}\text { Cumulative } \\
\text { Incidence (per 1000) }\end{array}$ & $\begin{array}{l}\text { Relative Risk }(95 \% \\
\text { CI) }\end{array}$ \\
\hline Grade 0 & 2 & 182 & 11.0 & $3.8(0.79-19.4)$ \\
Grade I & 5 & 115 & 43.9 & $12.5(2.6-60.0)$ \\
Grade II & 6 & 38 & 162.2 & \\
\hline
\end{tabular}

Table 4: Risk factors for developing GDM/DM in pregnancy

\begin{tabular}{llll}
\hline Factor & Adjusted OR & $\mathbf{9 5 \%}$ CI & Significance (p) \\
\hline Fatty liver grade II & 12.3 & $2.2-66.4$ & 0.003 \\
Fatty liver grade I & 3.3 & $0.6-18.7$ & 0.166 \\
Age & 0.9 & $0.3-2.2$ & 0.829 \\
Parity (primi over multi gravida) & 2.5 & $0.6-10.2$ & 0.191 \\
BMI & 1.4 & $0.7-2.8$ & 0.272 \\
Waist-to-hip ratio & 0.8 & $0.3-1.6$ & 0.579 \\
Family history of diabetes & 0.6 & $0.1-3.5$ & 0.642 \\
Female education & 0.7 & $0.5-1.1$ & 0.170 \\
\hline
\end{tabular}

Hosted file

Figures-FL_BJOG-final.docx available at https://authorea.com/users/420771/articles/527029non-alcoholic-fatty-liver-disease-nafld-as-a-major-predictor-of-gestational-diabetesmellitus-gdm-and-early-pregnancy-miscarriage-a-prospective-cohort-study 